\title{
Réduire les cas hautement déficitaires dans SwissDRG
}

\author{
Petra Ingenpass ${ }^{a}$, Barbara Rohner ${ }^{b}$ \\ a Dr med., cheffe adjointe de la division Tarifs et économie de la santé pour les médecins hospitaliers \\ b Collaboratrice scientifique de la division Tarifs et économie de la santé pour les médecins hospitaliers
}

Une représentativité pertinente des cas hautement déficitaires est inscrite depuis des années au programme d'amélioration de SwissDRG. Entre-temps, SwissDRG SA a examiné quatre propositions de solution et pris la décision de fond suivante: les solutions pour une rémunération adaptée aux dépenses des cas hautement déficitaires doivent être cherchées au sein de la structure tarifaire et inclure les cas de traitement bénéficiaires.

\section{Cas nettement déficitaires ou bénéficiaires}

SwissDRG est un système de rémunération forfaitaire qui regroupe des cas comparables du point de vue économique et médical, et qui les rémunère selon un forfait moyen par groupe de cas. Les écarts entre les recettes et le coût réel des ressources mises en œuvre font partie intégrante du système. Dans l'idéal, la part de cas bénéficiaires compense les cas déficitaires au sein d'un même hôpital. En réalité, les cas hautement déficitaires sont généralement répartis de manière asymétrique entre les hôpitaux et, selon SwissDRG SA, il est donc pratiquement impossible de compenser les cas déficitaires par des cas bénéficiaires. Cela vaut principalement pour les hôpitaux universitaires, les hôpitaux pour enfants ou les fournisseurs de prestations médicales extrêmement complexes. Les cas hautement déficitaires sont en effet le résultat de constellations particulières - les coûts de traitement sont tels que les ressources mises en œuvre sont loin d'être couvertes par la rémunération des forfaits par cas.

\section{Quatre propositions de solution discutées}

SwissDRG SA a tout à fait conscience du problème que posent les cas très coûteux et elle les a repris dans sa stratégie de développement 2013+. C'est pourquoi le groupe de travail de SwissDRG SA chargé des cas hautement déficitaires a présenté quatre propositions en vue d'une rémunération adaptée aux dépenses.

$1^{r e}$ proposition: au fil de ses développements annuels, la structure tarifaire SwissDRG doit faire l'objet d'une meilleure différenciation de sorte que les cas hautement déficitaires puissent, si possible, être évités. Pour cela, il faudrait d'une part revoir l'ensemble des règles de calcul, dont notamment l'analyse selon la différence des coûts, le mode de calcul des outliers et le calcul des coûts relatifs (cost-weight) exclusivement sur la base des coûts des inliers. D'autre part, il faut également regarder si les caractéristiques spécifiques aux cas hautement déficitaires peuvent être rémunérées par le biais de contributions additionnelles. Les cas hautement déficitaires sont fréquemment associés à une durée d'hospitalisation plus longue, à un transfert d'un autre hôpital, à des infirmités congénitales ou à un séjour en soins intensifs. Ces caractéristiques doivent être définies.

Proposition 2: cette proposition prévoit la différenciation du taux de base (baserate) des hôpitaux faisant état d'une part de cas hautement déficitaires supérieure à la moyenne, c.-à-d. les hôpitaux universitaires et les cliniques offrant des prestations spécialisées et complexes. Il s'agit donc de définir des critères objectifs pour la différenciation des taux de base en considérant non seulement le nombre de cas hautement déficitaires et le volume du déficit, mais aussi les cas hautement bénéficiaires afin d'éviter les distorsions. Si ces mesures devaient ne pas suffire pour contrer la concurrence déloyale des fournisseurs de prestations avec un taux de base plus élevé, il faudrait alors examiner des mesures supplémentaires.

S'agissant des solutions proposées en dehors de la structure tarifaire (comme c'est le cas des propositions 3 et 4 ci-après), des questions se posent au sujet de la convention tarifaire appliquée dans toute la Suisse et approuvée par le Conseil fédéral: quand parle-t-on de cas hautement déficitaire? Comment y est défini le financement additionnel? Quels critères doivent être remplis pour qu'un hôpital obtienne un financement additionnel de ses cas hautement déficitaires? S'agis- 
sant du financement additionnel, des dispositions ont été prises concernant la qualité des données, le volume maximum de rémunérations additionnelles ou encore la proportion de cas bénéficiaires par rapport aux cas déficitaires. Une couverture intégrale du déficit n'est cependant pas prévue, les hôpitaux doivent continuer de supporter eux-mêmes une partie de la sous-couverture. Les propositions de solution 3 et 4 provoqueraient une interaction entre le financement additionnel et le taux de base à négocier. De plus, ces deux variantes doivent également être examinées de point de vue de leur conformité avec la LAMal.

Proposition 3: chaque cas hautement déficitaire bénéficie d'une rémunération additionnelle de la part de ceux qui répondent des coûts. Le taux de couverture maximum est préalablement défini dans la convention de la structure tarifaire.

Proposition 4: cette proposition prévoit des versements à partir d'un pool grâce auquel tous les hôpitaux avec des cas hautement déficitaires peuvent faire valoir leurs prestations. Ce pool pourrait par exemple être alimenté par une contribution de solidarité sur chaque cas.

\section{La FMH plaide pour une meilleure différenciation de SwissDRG}

La FMH souhaite une rémunération adaptée aux dépenses des cas hautement déficitaires et soutient une approche visant à rechercher des solutions conformes à la LAMal au sein de la structure tarifaire SwissDRG. L'évolution annuelle de SwissDRG a déjà permis d'atteindre une meilleure représentativité de ces cas, notamment grâce à la création de davantage de contributions additionnelles. Pour la FMH, il faut continuer sur cette voie et rechercher une meilleure différenciation dans le cadre des possibilités structurelles. Pour cela, il importe de vérifier si les évolutions similaires du système G-DRG (le système allemand) sont utiles et compatibles avec SwissDRG. Par exemple, le système allemand a précisé et élargi son échelle de sévérité pour une meilleure représentativité de l'ensemble des cas les plus chers. ${ }^{1}$ Mais pour la FMH, des contributions additionnelles pour les caractéristiques spécifiques aux cas hautement déficitaires constituent aussi une approche pertinente qu'il s'agit donc de poursuivre. Avant que cette différenciation de la structure tarifaire, nécessaire pour les cas hautement déficitaires, produise ses effets, il est inévitable de passer par des taux de base différenciés. Dès l'introduction de SwissDRG, la FMH s'était déjà prononcée pour une solution transitoire en faveur de taux de base différenciés en fonction de la catégorie d'hôpital, dans le but de compenser une structure tarifaire encore insuffisante à l'époque. Leur utilisation prolongée ne résout cependant pas le cœur du problème. Au contraire, elle accentue la concurrence déloyale envers les hôpitaux qui ont un mandat de prestations pour la médecine hautement spécialisée très complexe. Enfin, les taux de base différenciés compliquent non seulement les négociations tarifaires mais en plus, conformément à l'arrêt du Tribunal administratif fédéral, ils ne sont conformes à la LAMal que pendant la phase d'introduction de SwissDRG. ${ }^{2}$

La majorité des sociétés de discipline médicale et des organisations faîtières estiment que la proposition 3 est trop complexe et que, par ailleurs, elle comporte encore beaucoup de questions sans réponse. Une fois que celles-ci auront été clarifiées par SwissDRG SA, il serait pertinent d'étudier l'opportunité de voir cette variante devenir une solution transitoire de remplacement jusqu'à ce que la structure soit mieux différenciée. En revanche, la solution via un pool comme le prévoit la proposition 4 devrait être difficilement applicable et donc plutôt abandonnée.

\section{SwissDRG SA poursuit la différenciation mais avec une définition problématique des cas hautement déficitaires}

Le conseil d'administration de SwissDRG SA s'est prononcé en faveur d'une meilleure différenciation des cas hautement déficitaires dans la structure tarifaire; une décision saluée par la FMH. Il a par ailleurs décidé que les cas hautement bénéficiaires soient aussi pris en compte et que les variantes élaborées en dehors de la structure tarifaire soient pour l'heure abandonnées. Dans la version 6.0 de SwissDRG, SwissDRG SA utilise une définition très restrictive des cas très coûteux. Sont réputés comme tels les cas dont les coûts totaux s'élèvent au moins à 400000 francs et présentent un déficit d'au moins 40000 francs. Une définition qui ne correspond qu'à quelques cas et qui est donc plus que problématique. Pour la FMH, les coûts totaux sont inadéquats, seul le déficit est pertinent dans cette définition.

Dès lors, il est d'autant plus important que SwissDRG SA informe des progrès faits en matière de rémunération des cas hautement déficitaires lors de la présentation annuelle de la nouvelle version de SwissDRG en montrant notamment l'impact des mesures prises et leurs effets provoqués jusqu'à présent. Dans ce contexte, il serait également important que SwissDRG SA rappelle les limites de la structure tarifaire en matière de représentativité des cas hautement déficitaires. Même si la voie choisie ne mène pas encore à une solution définitive, il est tout de même recommandé de la suivre avec détermination et rapidité.

Pour consulter la prise de position de la FMH sur SwissDRG et les cas hautement déficitaires ainsi que les mesures proposées, rendez-vous sur: www.fmh.ch $\rightarrow$ Tarifs hospitaliers $\rightarrow$ Positions $\rightarrow$ Prises de position. 